\title{
Análisis de ordenamiento de la movilidad en el bulevar gastronómico de Milán, Manizales (Colombia)
}

\author{
Santiago Cardona ${ }^{(1)}$, Diego A. Escobar ${ }^{(1) *}$ y Carlos A. Moncada(2) \\ (1) Universidad Nacional de Colombia, Sede Manizales. Facultad de Ingeniería y Arquitectura, \\ Departamento de Ingeniería Civil, Grupo de investigación en Movilidad Sostenible. Km 9 vía al aeropuerto La Nubia, \\ Bloque S2, Oficina 205 - 208, Manizales, 170003, Colombia. (correo-e: scardonau@ual.edu.co; \\ daescobarga@unal.edu.co) \\ (2) Universidad Nacional de Colombia, Sede Bogotá. Facultad de Ingeniería, Departamento de Ingeniería \\ Civil y Agrícola, Programa de Investigación en Tránsito y Transporte - PIT. Ciudad Universitaria edificio 214 \\ oficina 417, Bogotá, 111321, Colombia. (correo-e: camoncadaa@unal.edu.co)
}

* Autor a quien debe ser dirigida la correspondencia

Recibido Abr. 1, 2019; Aceptado May. 31, 2019; Versión final Jul. 29, 2019, Publicado Feb. 2020

\section{Resumen}

Se determina el impacto en el tiempo de viaje generado por el redireccionamiento vial propuesto en el sector gastronómico de Manizales, en Colombia. El estudio tiene como objetivo ayudar a solucionar o mejorar las problemáticas en movilidad existentes, tratando de eliminar la congestión vehicular generada por la baja oferta de parqueo y la alta demanda de usuarios. Estos factores dificultan el tránsito normal de los vehículos y peatones. El estudio se basa en el uso de la accesibilidad media global y accesibilidad integral, como elementos de cálculo y metodología de estimación del impacto generado por la intervención. Como resultado se obtiene que el $100 \%$ de la población podría acceder a la zona gastronómica en un tiempo de viaje inferior a los 45 minutos.

\section{Analysis of mobility reorganization of the gastronomic boulevard of Milan, Manizales (Colombia)}

\begin{abstract}
The impact on travel time generated by redirection of roads in the gastronomy sector of Manizales, Colombia is analyzed. The objective of the study is to help in solving or contributing to a solution of the problems of mobility, trying of eliminating traffic congestion, low parking offer and the high demand of users. These factors make it difficult the normal transit of vehicles and pedestrians. The study is based on the use of global average accessibility and integral accessibility, as elements of calculation and the methodology of estimating the impact generated by intervention. As a result, $100 \%$ of population could reach the gastronomy sector in a travel time of less than 45 minutes.
\end{abstract}

Keywords: accessibility; gastronomic zone; urban planning; road re-direction 


\section{INTRODUCCIÓN}

El impulso de las zonas gastronómicas, lugares donde se concentra un gran número de restaurantes en una ciudad, es parte fundamental para hacer de una ciudad un potencial gastronómico por excelencia, además de incrementar su atractivo turístico (Bahls et al., 2015). En este sentido, Manizales (Figura 1) capital del Departamento de Caldas, a través de su administración municipal, desde el año 2015 ha impulsado su propia zona gastronómica llamada "Zona G", ubicada en el barrio Milán de la ciudad, sector en el cual se han invertido más de 1,75 millones USD en mejoras urbanísticas que incluyen la construcción de un boulevard que contempla la ampliación de andenes, sitios de parqueo legal para vehículos livianos, demarcación de zonas de descarga, definición de ciclo-ruta y un mejoramiento en la iluminación pública.

La Alcaldía de Manizales unificó los sentidos viales en la Zona G con el fin de mejorar la movilidad y así ampliar las zonas de parqueo debido a que se estaba presentando una fuerte demanda contrastada con una poco oferta, lo que ocasionaba frecuentes maniobras de estacionamiento a lo largo de aceras y vía, que al ser doble sentido obstaculizaba enormemente el flujo vehicular (Centro de Prensa Alcaldía de Manizales, 2018a). Sin embargo, dicha medida tuvo resistencia en la comunidad residente del sector debido a que no se contaban con estudios técnicos que soportaran la decisión y no se cuantificaba la afectación o beneficio real para ellos junto a las medidas tomadas para mitigarlos. La Alcaldía determinó que la unificación iba a contar con una prueba piloto de 1 mes ( 5 de junio a 5 de julio de 2018) donde se buscaba medir el impacto en campo, generado por dichas unificaciones de sentido vial único (Centro de Prensa Alcaldía de Manizales, 2018b). Así mismo, se iban a realizar diversas mesas técnicas de socialización y cuantificación de impacto con diversos representantes del sector comercial, residencial, educativo, de culto y comercial (Centro de Prensa Alcaldía de Manizales, 2018b).

La prueba piloto fue extendida por 2 semanas más con el fin de evaluar el impacto generado al momento del ingreso a clases de las instituciones educativas cercanas según informó la prensa local. La intervención del sector involucró la recuperación de los espacios destinados para modos de transporte autónomos (caminata y bicicleta), ampliando el ancho de las aceras a 2,5 metros en promedio a lo largo de 1,9 km de longitud y estableciéndose también un bicicarril que comparte superficie de rodadura con el vehículo privado a lo largo de aproximadamente un kilómetro; en relación con las personas como movilidad reducida, se logaron adaptar 24 pasos para este tipo de desplazamientos, incluyendo la debida demarcación horizontal, vertical y superficie especial para personas ciegas, complementado esto con la construcción de pasos pompeyanos en los sitios de mayor afluencia peatonal.

La accesibilidad, es un modelo de oferta del transporte, mediante el cual se busca medir la capacidad de interacción que poseen los individuos o zonas de una ciudad hacia las diferentes oportunidades ofrecidas, utilizando un modo de transporte particular y con unas variables de impedancia definidas (Geurs y Ritsema, 2001; Hansen, 1959). Este modelo, ha tenido diversos tipos de medida que han sido desarrollados a través de los años, entre los cuales se encuentran las basadas en gravedad (Hansen, 1959; Olsson, 2016), las medidas topológicas (Xiao et al., 2017), medidas de distancia o tiempo (Beria et al., 2017) y medidas basadas en utilidades (Nassir et al., 2016), entre otras.
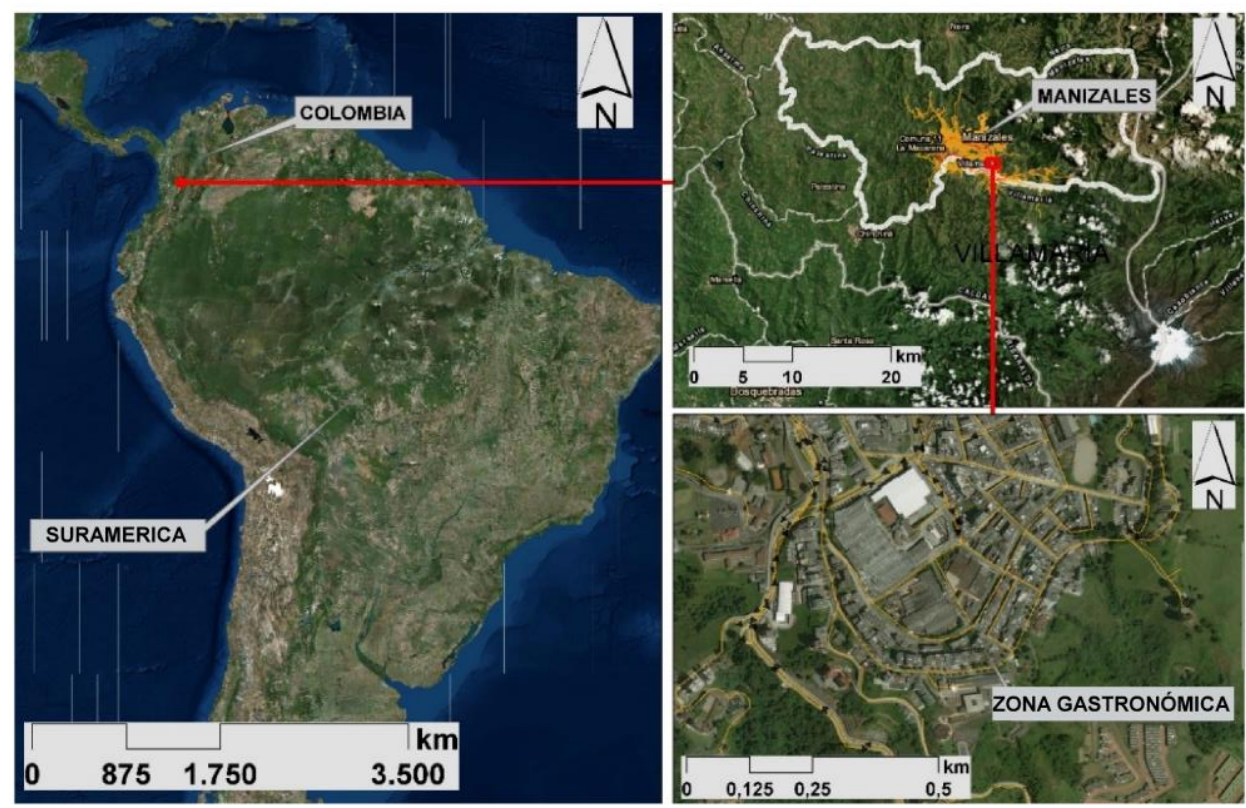

Fig. 1: Localización geográfica de la zona gastronómica de Manizales (Adaptada de googlemaps) 
Por otro lado, Ingram (1971) dividió el concepto de accesibilidad en dos tipos ampliamente conocidos, la accesibilidad relativa donde se realiza una medida entre dos puntos específicos de la ciudad y la accesibilidad integral donde se mide la accesibilidad de un conjunto de puntos respecto a otros o viceversa. Más adelante, en diversas investigaciones ha surgido el concepto de accesibilidad media global, donde se realiza una medida entre todos los nodos y se hace un análisis general de la oferta en la ciudad a través de la red de infraestructura vial, en diversos modos de transporte, como transporte público, transporte privado, bicicleta 0 caminata, según la velocidad promedio de cada uno de ellos (Escobar et al., 2018a). La accesibilidad media global, ha sido utilizada para evaluar el impacto generado por distintos cambios en la red de infraestructura vial (Escobar et al, 2018b) como la puesta en funcionamiento de nuevas obras de infraestructura vial como túneles y puentes (Moncada et al, 2018). Así mismo, la accesibilidad ha sido utilizada como método de planificación urbana (Mello y Portugal, 2017), usos del suelo (Geurs y van Wee, 2004), planificación del transporte público (Farber y Fu, 2017; Mavoa et al., 2012), acceso a servicios de salud (Rosero, 2004), estudios sobre exclusión social (Grengs, 2014), análisis de áreas metropolitanas (Allen et al, 1993; Escobar, et al, 2018a), acceso a librerías (Higgs et al., 2017), parques nacionales (Xiao et al., 2018) y trabajos (Geurs y Ritsema, 2003), entre muchos otros.

No se aborda un análisis de intervenciones peatonales y de bicicletas, pues claramente la construcción y recuperación realizada generó importantes beneficios en dicho aspecto, a pesar de las inconformidades presentadas por algunos colectivos comunitarios; es por ello que desde el punto de vista peatonal, las aceras se encuentran en un estado óptimo, tanto desde una mirada funcional como desde una mirada relacionado con su adaptación acertada para personas con movilidad reducida. Por otra parte, la comunidad de comerciantes del sector, a pesar que al comienzo del proyecto estuvieron en contra del mismo, en la actualidad han comprendido y comprobado que tienen mayores beneficios al haberse recuperado el sector dándole prioridad a modos de transporte autónomo, lo cual atrae flujos de caminantes que ingresan a disfrutar los productos que en dichos establecimientos se venden. En este artículo investigativo, se busca medir el impacto que genera la unificación vial en la Zona $G$ de la ciudad en los tiempos medios de viaje de los habitantes mediante la cuantificación del gradiente de ahorro en la accesibilidad media global de Manizales y la accesibilidad media integral de los ciudadanos que desean acceder en modo de transporte vehículo privado a la Zona G, comprendiendo de antemano que las condiciones para los modos de transporte autónomos se encuentran adecuadamente solventadas para un radio de influencia moderado.

\section{METODOLOGÍA}

La metodología se compone de cuatro fases principales, incluida una etapa de recolección y entrada de datos necesarios para realizar los cálculos y análisis (Figura 2).

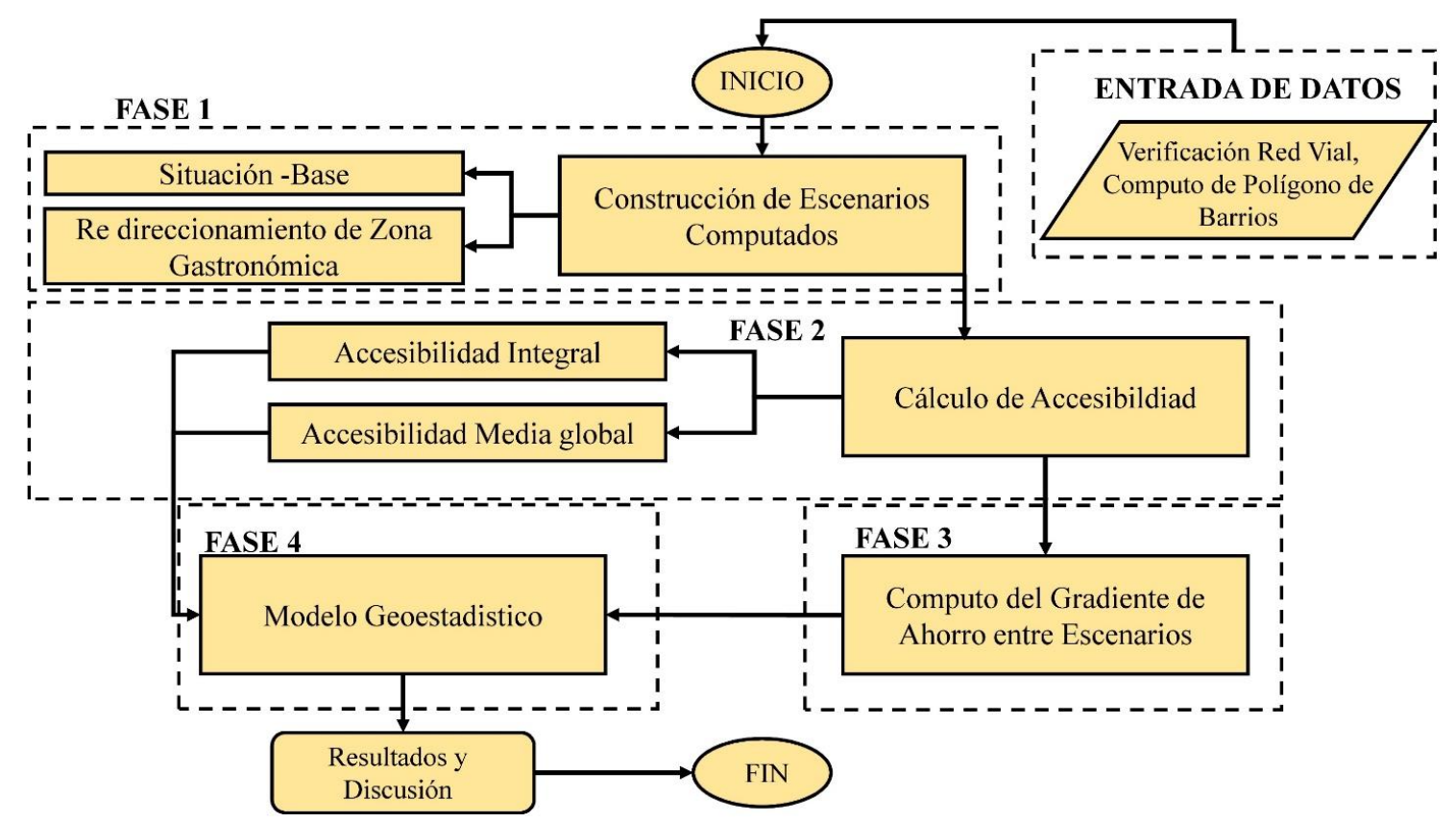

Fig. 2: Metodología de investigación abordada

\section{Fase 1. Red vial y polígono de barrios}

La red de infraestructura vial para la zona de estudio se obtiene por medio de investigaciones previas (Moncada et al., 2018), las cuales poseen las últimas obras de infraestructura vial inauguradas en la ciudad, 
así mismo poseen las condiciones necesarias para realizar los cálculos posteriores, entre las que se enumeran cumplir con la teoría de grafos que incluye la construcción de la red mediante nodos y arcos (Kozyrev, 1972), las velocidades de los arcos de la red y el cálculo de las longitudes y tiempos de viaje por arco mediante herramientas GIS; se resalta que el dato de velocidades de operación se toma desde estudios oficiales como el Plan Maestro de Movilidad de la ciudad (Escobar y García, 2012), para el cual se calcularon las velocidades promedio de los arcos a partir de datos tomados durante un período de más de un año, estableciéndose que la presente investigación no se relaciona con algunos días específicos de estudio, sino con un día típico y promedio, es decir, se relaciona con las velocidades que más probablemente se pueden encontrar un día considerado común o típico en la ciudad. Por otra parte, es necesario tomar el polígono de barrios de Manizales (Moncada et al., 2018) y actualizar los datos correspondientes a la población para 2018 mediante las proyecciones de población del Departamento Administrativo Nacional de Estadística (DANE, 2010). Se destaca que tanto la red vial como los polígonos de barrios son dos capas de información, no sólo fundamentales para los cálculos, sino también información que podría ser adquirida en otras poblaciones con el fin de replicar los cálculos presentados en esta investigación; la red vial, por lo general, es posible obtenerla a través de la secretaría de tránsito o movilidad que exista, o en un caso extremo, mediante su elaboración a partir de herramientas como googlemaps; así mismo, la capa de información sociodemográfica de barrios es posible conseguirla a través de la secretaría de planeación o mediante estadísticas municipales oficiales, Planes de Ordenamiento Territorial, entre otros documentos.

En esta investigación se tuvieron en cuenta dos escenarios: i) Cálculos de accesibilidad con los sentidos que presenta la red de infraestructura vial antes de los cambios de dirección en la Zona G, denominado situación base; ii) Cálculo de la accesibilidad incluyendo los cambios de sentido presentes en la zona de estudio, denominado redirecciones zona gastronómica (Centro de Prensa Alcaldía de Manizales, 2018b). Para el cálculo del escenario futuro en la accesibilidad media global y accesibilidad media integral se deben delimitar los nodos de la red de infraestructura vial que hacen parte de la zona gastronómica y realizar los cambios de dirección adoptados por la Alcaldía de Manizales (Centro de Prensa Alcaldía de Manizales, 2018a). En la parte A de la Figura 3 se encuentra la red de infraestructura vial con los cambios de dirección sugeridos y realizados por la Alcaldía de Manizales, donde fundamentalmente se unificaron los sentidos de 3 vías sobre la zona de estudio, generando que el flujo realice un movimiento en sentido contrario a las manecillas del reloj para entrar y salir de la zona. En la parte B de la Figura 3, se observa la dirección actual que posee la Zona G, que corresponde al escenario base de cálculo (vías bidireccionales o doble sentido). Esta fase metodológica es totalmente replicable siempre y cuando se posean la capa de información de la red vial, la cual es base para realizar las modificaciones pertinentes, y que se desean evaluar, para construir el escenario futuro, dado lo anterior, se considera que la haberse superado la fase anterior, ésta es totalmente factible para aplicarse en una investigación similar para otra población.
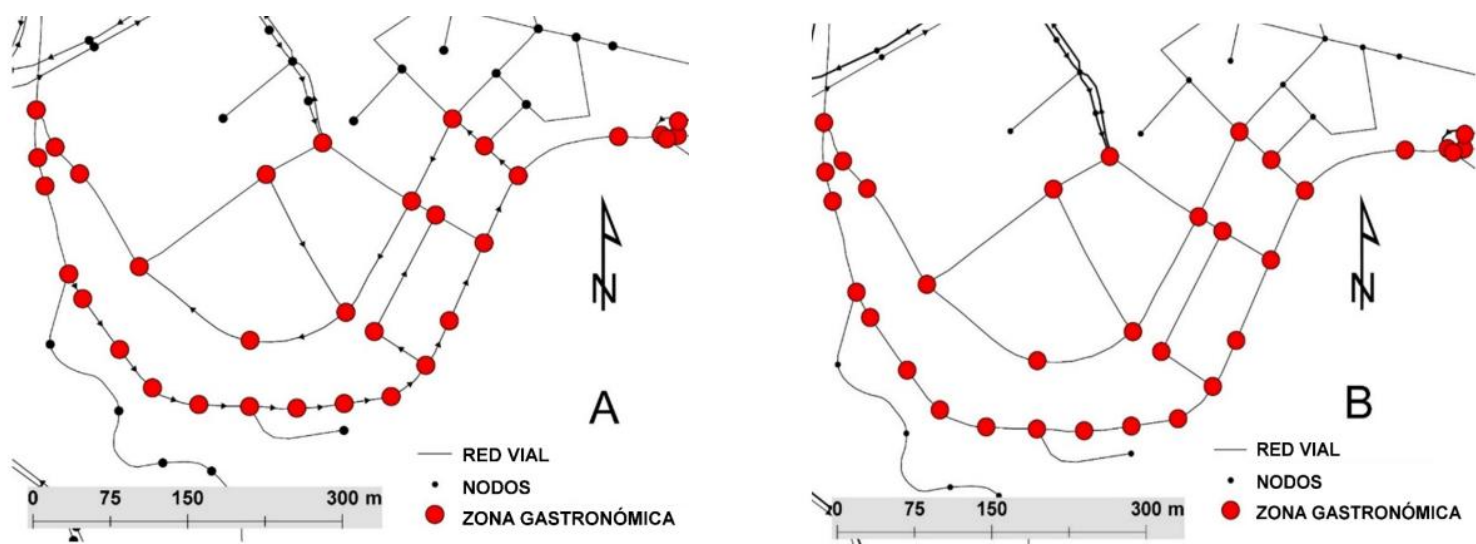

Fig. 3: Red de infraestructura vial. A) Re direccionamiento de la zona gastronómica. B) Situación base.

\section{Fase 2. Cálculo de accesibilidad}

En esta fase se procede a calcular la accesibilidad media global y accesibilidad media integral para ambos escenarios; al ser ambos métodos de análisis de redes, y al haberse superado las dos fases anteriores, es factible el replicar los cálculo para otras investigaciones geográficas similares. Para la obtención de las curvas isócronas de accesibilidad se debe aplicar el algoritmo Djkstra (1959), el cual se encuentra incluido en la extensión Network Analyst de ArcMap y mediante el cual se calcula el vector de tiempo de viaje (Tvi) desde los nodos que conforman la red vial (i) hacia cada una de las facilidades (j) (Ver Expresión (1)).

$$
\overline{T_{v l}}=\frac{\sum_{j=1}^{n} t_{v i}}{(n-1)} \quad i=1,2,3, \ldots, n ; j=1,2,3, \ldots, \mathrm{n}
$$


Luego de obtenido el vector de tiempo medio de viaje, se calcula la matriz de tiempos promedios mínimos de viaje, a la cual se le correlacionan las coordenadas de cada nodo de la red, llegando a una matriz de dimensión ( $\mathrm{n} \times 3$ ) con la cual se construyen las curvas isócronas en ambos tipos de accesibilidad. El procedimiento de cálculo de la accesibilidad, en ambos casos, es totalmente replicable siempre y cuando se tengan las dos primeras fases superadas y se posea la red vial cargada con las características operativas (velocidad de operación) para lograr calcular los tiempos medios de viaje. Una vez calculadas las curvas isócronas, se correlacionan éstas con la capa de información sociodemográfica (población y área de la zona de estudio), para determinar los porcentajes de cobertura para cada escenario y para cada tipo de accesibilidad estudiado.

El cálculo de la accesibilidad media global es un promedio de los tiempos medios de viaje entre todos los nodos de la red vial. Posteriormente, mediante el software TRANSCAD 8.0, software GIS especializado en modelos de transporte, se corre el algoritmo de Dijkstra de caminos mínimos (Dijkstra, 1959) para encontrar la mejor ruta posible entre todos los nodos y así computar la matriz de tiempos medios de viaje. Posteriormente se realiza la sumatoria de tiempos para cada nodo y se divide entre los el número total de nodos de la red, obteniendo el vector de tiempo promedio de viaje, insumo necesario para la construcción de las curvas isócronas de accesibilidad mediante el modelo geoestadístico (Moncada et al., 2018). Para el cálculo de la accesibilidad media integral se deben definir los nodos que corresponden a la Zona G. Luego de esto se realiza el cálculo de la matriz de tiempos de viaje de todos los nodos de la red vial hasta los nodos objetivo, de la misma forma que se hizo en la accesibilidad media global, utilizando el software TRANSCAD 8.0 y el algoritmo de caminos mínimos (Dijkstra, 1959). Por último, se obtiene el vector de tiempos de accesibilidad integral, haciendo la sumatoria de los tiempos de cada nodo y dividiendo entre el número de nodos objetivo. Este vector, es usado en el modelo geoestadístico y obtener las curvas isócronas de accesibilidad media integral.

\section{Fase 3. Cálculo del Gradiente de ahorro entre escenarios}

El cálculo del gradiente de ahorro se utiliza para confrontar dos escenarios de accesibilidad, en este caso la accesibilidad media global y accesibilidad media integral del escenario base y el de redirecciones de la Zona G. Para ello se utilizan los vectores de tiempo medio de viaje obtenidos en la etapa anterior y se calcula el gradiente de ahorro (Escobar et al., 2018a). Resultando un vector de porcentaje de ahorro de tiempos medios de viaje que es utilizado para aplicar el modelo geoestadístico y así determinar cuáles fueron las zonas afectadas o beneficiadas por las medidas tomadas en cuanto a redireccionamiento en la Zona G (Escobar et al., 2018a).

\section{Fase 4. Modelo geoestadístico}

Con los vectores de tiempo medio de viaje de la accesibilidad media global e integral y el vector de gradiente de ahorro se procede a aplicar el modelo geoestadístico Kriging ordinario con semivariograma lineal como predictor de valores de tiempo medio de viaje para zonas donde no se conocían datos de entrada y lograr así calcular las curvas isócronas (Simpson et al., 2001), este modelo evalúa la correlación espacial entre variables definidas, permitiendo la generación de superficies continuas a partir de nodos conocidos; con el semivariograma se evalúan las propiedades de dependencia espacial entre nodos de la red vial estudiada, obtenido mediante la Expresión (2), donde $Z_{(x)}=$ valor de la variable tiempo medio de viaje en un sitio con coordenadas $x, y ; Z_{(x+h)}=$ valor muestral de otro punto separado por una distancia $h ; n=$ número de parejas identificadas que se encuentran separadas por la misma distancia $h$. Dicho modelo ha sido utilizado a lo largo de los años como modelo predictor de modelos de oferta de transporte, siendo éste uno de los más utilizados por diversos investigadores en el campo (Escobar et al., 2018a).

$$
\overline{\gamma_{(h)}}=\frac{\sum\left(Z_{(x+h)}-Z_{(x)}\right)^{2}}{2 n}
$$

\section{RESULTADOS Y DISCUSIÓN}

La ejecución de la metodología formulada, permitió obtener los resultados que se presentan en dos subsecciones: (i) Accesibilidad Media Global y gradiente de ahorro; y (ii) Accesibilidad media integral y gradiente de ahorro.

\section{Accesibilidad Media Global y Gradiente de Ahorro}

En la Figura 4 se observan las curvas isócronas de accesibilidad media global para el escenario base (parte izquierda) y para el escenario con la redirección de la Zona G (parte derecha), donde el tiempo mínimo y máximo registrado para los tiempos promedio de viaje es igual con 16,59 minutos y 52,11 minutos respectivamente por lo que ambos escenarios son similares en este sentido. Así mismo, en el recuadro donde 
se realiza un acercamiento a la zona de estudio, se nota un pequeño cambio en las curvas isócronas, donde la curva isócrona entre 16,59 minutos y 20 minutos disminuye su zona de influencia en el escenario de re direccionamiento de la Zona $\mathrm{G}$, dado que la curva isócrona entre 20 y 25 minutos aumentó.
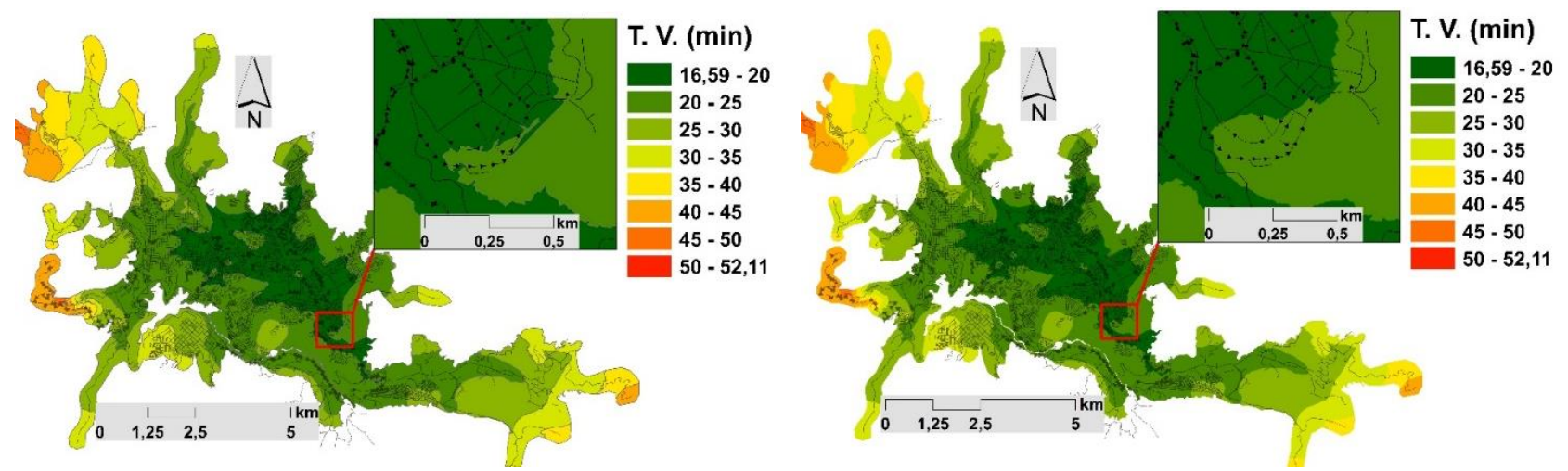

Fig. 4: Curvas isócronas, accesibilidad media global

El análisis de cobertura y población para ambos escenarios de las curvas isócronas de accesibilidad media global se presenta en la Tabla 1 y la Tabla 2. Se observa cómo la cobertura de población y área para la curva isócrona entre 16,59 y 20 minutos disminuye en $0,13 \%$ ( 557 habitantes y 8 ha) entre el escenario base y el escenario de re direccionamiento de la zona gastronómica, sumándose a la cobertura de la siguiente curva isócrona. En las demás curvas isócronas no se parecía una diferencia significativa. Por último, cabe destacar que el $100 \%$ de la población se encuentra cubierta por un tiempo máximo de hasta 45 minutos, lo que nos indica que la densidad poblacional de la ciudad se concentra en un área menor a la potencial y existen polígonos analizados donde no habitan mayor número de personas ya que corresponden a las partes periféricas, de salida a otras ciudades y zonas de expansión (Escobar y García, 2012).

Tabla 1: Cobertura por habitantes y área, accesibilidad media global - Situación Base

\begin{tabular}{|c|c|c|c|c|c|c|}
\hline Curva Isócrona & Área & Habitantes & $\%$ Área & $\%$ Habitantes & $\%$ Área Acumulada & \%Habitantes Acumulados \\
\hline $16,59-20$ & 876,51 & $134.081,83$ & $14,95 \%$ & $31,95 \%$ & $14,95 \%$ & $31,95 \%$ \\
\hline $20-25$ & $2.091,29$ & $209.225,44$ & $35,67 \%$ & $49,85 \%$ & $50,61 \%$ & $81,80 \%$ \\
\hline $25-30$ & $1.389,99$ & $57.039,11$ & $23,71 \%$ & $13,59 \%$ & $74,32 \%$ & $95,39 \%$ \\
\hline $30-35$ & 746,14 & $15.298,88$ & $12,72 \%$ & $3,65 \%$ & $87,04 \%$ & $99,04 \%$ \\
\hline $35-40$ & 406,17 & $2.960,65$ & $6,93 \%$ & $0,71 \%$ & $93,97 \%$ & $99,74 \%$ \\
\hline $40-45$ & 258,14 & $1.080,92$ & $4,40 \%$ & $0,26 \%$ & $98,37 \%$ & $100,00 \%$ \\
\hline $45-50$ & 57,30 & 0,00 & $0,98 \%$ & $0,00 \%$ & $99,35 \%$ & $100,00 \%$ \\
\hline $50-52,11$ & 38,10 & 0,00 & $0,65 \%$ & $0,00 \%$ & $100,00 \%$ & $100,00 \%$ \\
\hline Total & $5.863,63$ & $419.686,84$ & $100 \%$ & $100 \%$ & & \\
\cline { 1 - 2 } & &
\end{tabular}

Tabla 2: Cobertura por habitantes y área, accesibilidad media global - Escenario Futuro

\begin{tabular}{|c|c|c|c|c|c|c|}
\hline Curva Isócrona & Área & Habitantes & $\%$ Área & $\%$ Habitantes & $\%$ Área Acumulada & $\%$ Habitantes Acumulados \\
\hline $16,59-20$ & 868,26 & $133.484,54$ & $14,81 \%$ & $31,81 \%$ & $14,81 \%$ & $31,81 \%$ \\
\hline $20-25$ & $2.099,33$ & $209.782,03$ & $35,80 \%$ & $49,99 \%$ & $50,61 \%$ & $81,79 \%$ \\
\hline $25-30$ & $1.390,04$ & $57.079,72$ & $23,71 \%$ & $13,60 \%$ & $74,32 \%$ & $95,39 \%$ \\
\hline $30-35$ & 746,20 & $15.298,94$ & $12,73 \%$ & $3,65 \%$ & $87,04 \%$ & $99,04 \%$ \\
\hline $35-40$ & 406,28 & $2.960,55$ & $6,93 \%$ & $0,71 \%$ & $93,97 \%$ & $99,74 \%$ \\
\hline $40-45$ & 258,13 & $1.081,04$ & $4,40 \%$ & $0,26 \%$ & $98,37 \%$ & $100,00 \%$ \\
\hline $45-50$ & 57,30 & 0,00 & $0,98 \%$ & $0,00 \%$ & $99,35 \%$ & $100,00 \%$ \\
\hline $50-52,11$ & 38,10 & 0,00 & $0,65 \%$ & $0,00 \%$ & $100,00 \%$ & $100,00 \%$ \\
\hline Total & $5.863,63$ & $419.686,80$ & $100 \%$ & $100 \%$ & & \\
\cline { 1 - 2 } & &
\end{tabular}

Por otra parte, en la Figura 5 se aprecia el gradiente de ahorro que analiza la diferencia en los tiempos de viaje dados en la accesibilidad media global de ambos escenarios estudiados; se observan diferencias en los tiempos medios de viaje de hasta $-4,2 \%$, concentrados cerca de la zona de estudio, donde incluso se observan beneficios de hasta $0,5 \%$. La mayor parte de la ciudad está cubierta por diferencias entre $-0,5 \%$ y $0 \%$, lo que nos indica que a nivel de accesibilidad media global no se genera un impacto importante sobre las condiciones de accesibilidad, las mayores diferencias se aprecian en el recuadro, que corresponde a la zona de estudio. 
Los resultados permiten establecer que el 99,45\% de la población (417.406 habitantes) y el 99,43\% del área (5.830 ha) se encuentran en una zona que refiere la inexistencia de ahorro en tiempo medio de viaje desde el punto de vista de accesibilidad media global, lo cual representa que el $0,16 \%$ de la población (669 habitantes) y $0,13 \%$ (8 ha) del área refieren un pequeño ahorro de tiempo medio de viaje en relación con el escenario base. Lo anterior indica que existen pocas diferencias entre ambos escenarios al evaluar el redireccionamiento y ordenamiento vial propuesto, no obstante, otras investigaciones realizadas en la ciudad, en las que se aplica la accesibilidad media global, han permitido encontrar importantes diferencias entre escenarios estudiados al evaluar el impacto generado por la inserción de infraestructura como rotondas, intercambiadores, túneles, etc. (Escobar et al., 2018b). Lo anterior significa que a pesar que las modificaciones en la direccionalidad de las vías del sector no registran un claro impacto sobre los tiempos medios de viaje, dicha intervención sí redundará en una disminución de las velocidades de operación y muy probablemente en la disminución de los índices de accidentalidad en el sector, lo cual coincide con la hipótesis planteada por Xiao et al (2017) en el sentido que existe un vínculo entre el uso del suelo y la accesibilidad, permitiendo develar características específicas en la decisión dela intervenciones a realizarse.

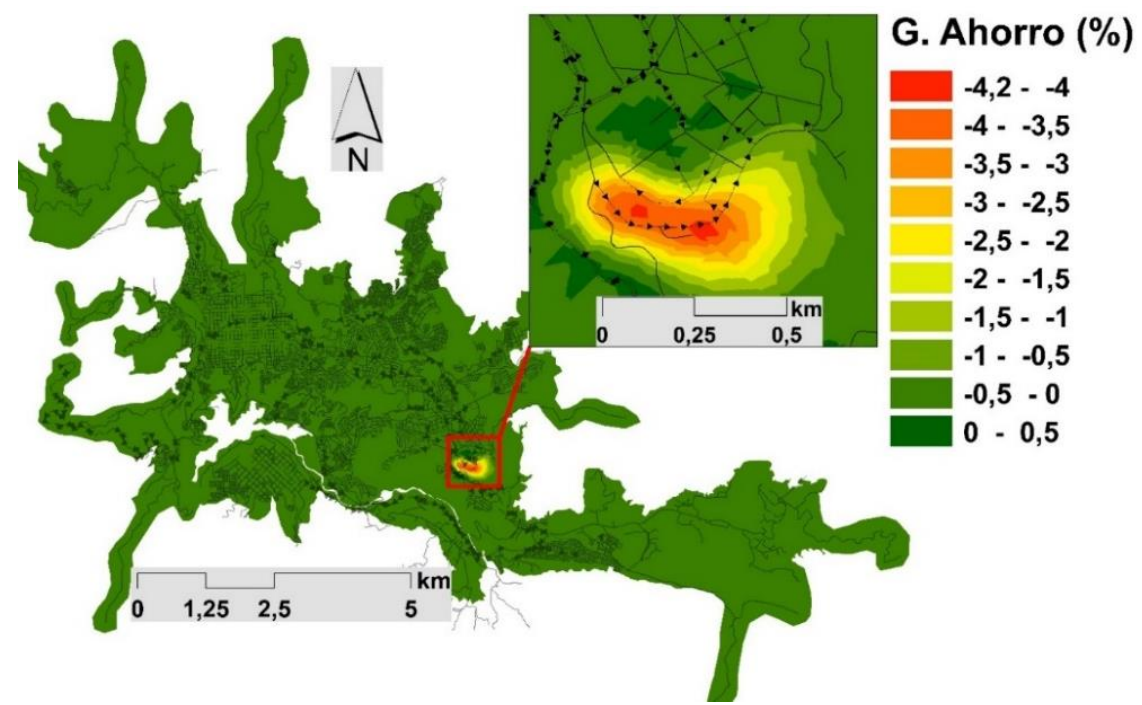

Fig. 5: Gradiente de ahorro, accesibilidad media global.

\section{Accesibilidad Media Integral y Gradiente de Ahorro}

En cuanto al resultado accesibilidad media integral para los escenarios estudiados, en la Figura 6 se observa que existe un tiempo de viaje mínimo a los nodos de la zona de estudio de 1,61 minutos en el escenario base (parte izquierda), mientras que para el escenario correspondiente al redireccionamiento de la Zona G (parte derecha), este valor aumenta hasta 2,43 minutos, explicado por la disminución de alternativas de ruta ocasionado por el cambio de las direcciones en los arcos, donde se pasa de doble sentido a un solo sentido. En cuanto los tiempos máximos, que se encuentran en las zonas al occidente de la ciudad, la diferencia en los tiempos es mínima con tan solo 0,06 minutos. Así mismo, en el recuadro se puede observar el cambio ocasionado en el tamaño de las curvas isócronas, donde la cobertura del primer intervalo de las curvas isócronas disminuye en el escenario del redireccionamiento, aumentando la cobertura de los siguientes intervalos de tiempo.
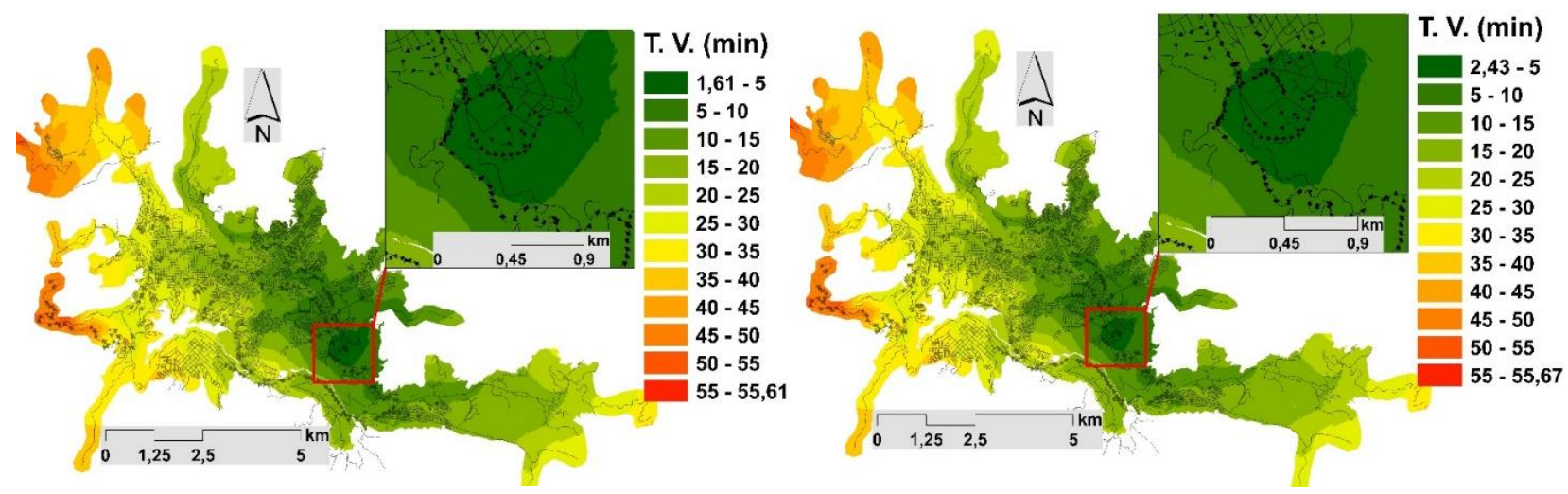

Fig. 6: Curvas isócronas, accesibilidad integral. 
En la Tabla 3 y la Tabla 4, se presenta de forma más detallada el análisis de cobertura de las curvas isócronas de accesibilidad media integral para la situación base y el escenario futuro, respectivamente, respecto a la población y área de la ciudad, en las cuales se observa una disminución, menor al 1\%, en la cobertura de las curvas isócronas, por lo que no existe un impacto sobresaliente en las condiciones de accesibilidad de la ciudad, pero apreciable en la zona de estudio. Al comprar los resultados obtenidos con otras investigaciones en las que se ha aplicado la metodología de accesibilidad media integral, y que han abordado el análisis de usos del suelo, se tiene que para este caso, la zona $\mathrm{G}$ tiene una cobertura del $80 \%$ de la población si se invierte en promedio unos 28 minutos de tiempo medio de viaje, valor inferior al encontrado para el análisis de los eco parques naturales (Escobar et al., 2018a), caso para el cual se encontró que para cubrir el mismo porcentaje de población se debería invertir hasta 30 minutos de tiempo medio de viaje, pero valor superior al encontrado cunado se analizó el uso del suelo relacionado con los centros comerciales, caso para el cual dicho valor de tiempo fue de 25 minutos de tiempo medio de viaje (Montoya et al., 2017).

Lo anterior indica que para el caso de la ciudad de Manizales, dadas las condiciones operativas de la red de infraestructura vial y la ubicación de la población, la localización en conjunto de los centros comerciales es más eficiente que la de la zona $G$ y la de los Ecoparques naturales, lo cual será positivo o negativo dependiendo de las políticas de desarrollo que se promuevan en la ciudad. Este tipo de análisis se configura como soporte base para el desarrollo de un estudio de Mercadeo, en relación con cualquier tipo de uso del suelo a implantarse, garantizando mayor cobertura según una población objetivo; por ejemplo, Zuluaga y Escobar (2017), encontraron que las personas que residen en barrios de alta capacidad económica tienen condiciones de accesibilidad más favorables a los centros comerciales que las personas que habitan barrios de baja capacidad económica.

Tabla 3: Cobertura por habitantes y área, accesibilidad media integral - Situación Base

\begin{tabular}{|c|c|c|c|c|c|c|}
\hline Curva Isócrona & Área & Habitantes & $\%$ Área & $\%$ Habitantes & $\%$ Área Acumulada & $\%$ Habitantes Acumulados \\
\hline $1,61-5$ & 76,31 & $5.879,38$ & $1,30 \%$ & $1,40 \%$ & $1,30 \%$ & $1,40 \%$ \\
\hline $5-10$ & 458,00 & $25.323,88$ & $7,81 \%$ & $6,03 \%$ & $9,11 \%$ & $7,43 \%$ \\
\hline $10-15$ & 920,15 & $109.862,17$ & $15,69 \%$ & $26,18 \%$ & $24,81 \%$ & $33,61 \%$ \\
\hline $15-20$ & $1.112,21$ & $76 . .025,26$ & $18,97 \%$ & $18,11 \%$ & $43,77 \%$ & $51,73 \%$ \\
\hline $20-25$ & $1.037,67$ & 96784,95 & $17,70 \%$ & $23,06 \%$ & $61,47 \%$ & $74,79 \%$ \\
\hline $25-30$ & 818,56 & $77.526,00$ & $13,96 \%$ & $18,47 \%$ & $75,43 \%$ & $93,26 \%$ \\
\hline $30-35$ & 628,45 & $23.524,04$ & $10,72 \%$ & $5,61 \%$ & $86,15 \%$ & $98,86 \%$ \\
\hline $35-40$ & 355,29 & $2.072,83$ & $6,06 \%$ & $0,49 \%$ & $92,21 \%$ & $99,36 \%$ \\
\hline $40-45$ & 207,34 & $2.155,72$ & $3,54 \%$ & $0,51 \%$ & $95,74 \%$ & $99,87 \%$ \\
\hline $45-50$ & 163,71 & 536,58 & $2,79 \%$ & $0,13 \%$ & $98,54 \%$ & $100,00 \%$ \\
\hline $50-55$ & 79,95 & 0,00 & $1,36 \%$ & $0,00 \%$ & $99,90 \%$ & $100,00 \%$ \\
\hline $55-55,61$ & 5,93 & 0,00 & $0,10 \%$ & $0,00 \%$ & $100,00 \%$ & $100,00 \%$ \\
\hline Total & 5863,58 & 419690,81 & $100 \%$ & $100 \%$ & & \\
\cline { 1 - 2 } & & &
\end{tabular}

Tabla 4: Cobertura por habitantes y área, accesibilidad media integral - Escenario Futuro

\begin{tabular}{|c|c|c|c|c|c|c|}
\hline Curva Isócrona & Área & Habitantes & $\%$ Área & $\%$ Habitantes & $\%$ Área Acumulada & $\%$ Habitantes Acumulados \\
\hline $2,43-5$ & 53,81 & $4.813,33$ & $0,92 \%$ & $1,15 \%$ & $0,92 \%$ & $1,15 \%$ \\
\hline $5-10$ & 437,20 & $22.267,70$ & $7,46 \%$ & $5,31 \%$ & $8,37 \%$ & $6,45 \%$ \\
\hline $10-15$ & 911,29 & $107.918,04$ & $15,54 \%$ & $25,71 \%$ & $23,92 \%$ & $32,17 \%$ \\
\hline $15-20$ & $1.128,50$ & $79.955,44$ & $19,25 \%$ & $19,05 \%$ & $43,16 \%$ & $51,22 \%$ \\
\hline $20-25$ & $1.049,01$ & $96 . .693,85$ & $17,89 \%$ & $23,04 \%$ & $61,05 \%$ & $74,26 \%$ \\
\hline $25-30$ & 833,49 & 79169,70 & $14,21 \%$ & $18,86 \%$ & $75,27 \%$ & $93,12 \%$ \\
\hline $30-35$ & 631,06 & $24.087,98$ & $10,76 \%$ & $5,74 \%$ & $86,03 \%$ & $98,86 \%$ \\
\hline $35-40$ & 358,42 & $1.991,59$ & $6,11 \%$ & $0,47 \%$ & $92,14 \%$ & $99,33 \%$ \\
\hline $40-45$ & 209,44 & 2234,94 & $3,57 \%$ & $0,53 \%$ & $95,71 \%$ & $99,87 \%$ \\
\hline $45-50$ & 163,59 & 560,11 & $2,79 \%$ & $0,13 \%$ & $98,50 \%$ & $100,00 \%$ \\
\hline $50-55$ & 81,14 & 0,00 & $1,38 \%$ & $0,00 \%$ & $99,89 \%$ & $100,00 \%$ \\
\hline $55-55,67$ & 6,64 & 0,00 & $0,11 \%$ & $0,00 \%$ & $100,00 \%$ & \\
\hline Total & $5.863,58$ & $419.692,69$ & $100 \%$ & $100 \%$ & & \\
\cline { 1 - 2 } & & & &
\end{tabular}

En cuanto al gradiente de ahorro, comparación de los escenarios en la accesibilidad media integral, su resultado se observa en la Figura 7 donde las mayores diferencias se aprecian en la zona gastronómica y sus alrededores, con intervalos entre $-106,29 \%$ y $-7,5 \%$ debido en gran medida a que los nodos utilizados para realizar el cálculo de la accesibilidad integral se encuentran sobre dicha zona y los cambios en los tiempos 
de viaje son mínimos para ambos escenarios por lo que una restricción en el direccionamiento vial producirá grandes diferencias en los mismos ya qué en el escenario futuro se deben hacer rutas más largas para viajar dentro de la Zona G. Así mismo, los gradientes de ahorro disminuyen en gran parte de la ciudad hasta porcentajes entre $-2,5 \%$ y $0 \%$, curva isócrona que posee mayor cobertura. En la zona nororiente de la ciudad y norte de la zona de estudio se perciben diferencias entre $-10 \%$ y $-2,5 \%$, debido a la cercanía a la Zona $G$ y a la disposición de los cambios en la dirección en sentido contrario a las manecillas del reloj.

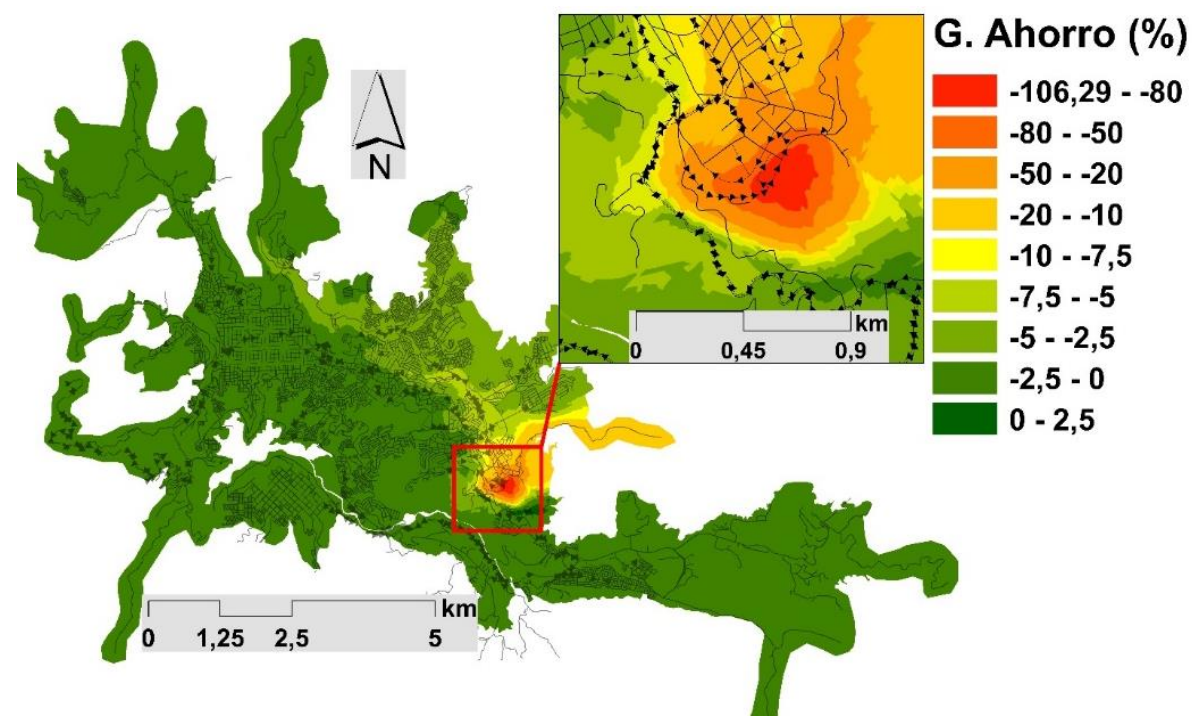

Fig. 7: Gradiente de ahorro, accesibilidad integral

\section{CONCLUSIONES}

En términos de accesibilidad media global, los impactos generados por la redirección vial en la zona gastronómica son mínimos en términos de tiempo medio de viaje, mientras que en la accesibilidad integral a la Zona G se generan unas diferencias apreciables en el área de estudio y la zona norte de la ciudad. Estos cambios de dirección pueden aumentar los tiempos de viaje en una gran parte de la ciudad, pero en porcentajes muy pequeños, en ambas medidas, debido a que los vehículos deben realizar rutas más largas para llegar a los mismos nodos dadas las unificaciones en la Zona G. Estás restricciones pueden generar una disminución en el uso del vehículo privado para dirigirse a la Zona G, generándose un aumento en las personas que acceden a ella mediante transporte público o la decisión de parquear el vehículo a sus alrededores y desplazarse dentro de ella caminando. Paralela a la unificación de dichas vías en la zona gastronómica se debe realizar un análisis de parqueo de vehículos sobre esta zona y sus alrededores con el fin de disminuir el parqueo en la vía e impulsar el parqueo en zonas autorizadas a los alrededores generando que el principal modo de transporte dentro de la zona sea la caminata.

Como conclusión final, este tipo de medida resulta beneficiosa en términos de movilidad debido a que contribuye al ordenamiento del tránsito diario. Sin embargo, este tipo de acciones deben ir de la mano con acciones urbanísticas y organizativas con el fin de impulsar medios de transporte sostenibles en la zona como el transporte público y la caminata con el fin de disminuir los problemas de parqueo del vehiculó privado y así hacer de la Zona G, en Manizales un lugar prioritario para la visita de los turistas y residentes de la ciudad.

\section{AGRADECIMIENTOS}

Los autores agradecen al grupo de investigación en movilidad sostenible de la Universidad Nacional de Colombia sede Manizales.

\section{REFERENCIAS}

Allen, W. B., D. Liu y S. Singer. Accesibility measures of U.S. metropolitan areas, doi:10.1016/0191-2615(93)90016-4, Transportation Research Part B, 27(6), 439-449 (1993).

Bahls, Á., R. W. Krause y F. Farias. Planejamento Gastronômico em Destinos Turísticos: Uma Comparação entre o Panorama Nacional e o Estrangeiro, Revista Rosa Dos Ventos - Turismo e Hospitalidade, ISSN: 2178-9061, 7(2), 223241 (2015).

Beria, P., A. Debernardi y E. Ferrara. Measuring the long-distance accessibility of Italian cities, doi:10.1016/j.jtrangeo.2017.05.006, Journal of Transport Geography, 62, 66-79 (2017). 
Centro de Prensa Alcaldía de Manizales. A partir del 5 de Junio habrá unificación vial en Milán. saladeprensamanizales.com (2018a).

Centro de Prensa Alcaldía de Manizales. Plan piloto de unificación vial para la zona G de Milán. saladeprensamanizales.com (2018b).

Departamento Administrativo Nacional de Estadística - DANE. Estimación y proyección de la población nacional, departamental y municipal por área 1985-2020 (2010).

Dijkstra, E. W. A note on two problems in connexion with graphs, doi:10.1007/BF01386390. Numerische Mathematik, 1(1), 269-271 (1959).

Escobar, D., y F. García. Diagnóstico de la Movilidad Urbana de Manizales (1 ${ }^{\text {era }}$ Ed). Manizales (2012).

Escobar, D., J. Montoya y C. Moncada. Accessibility Analysis towards Urban Ecoparks as a Measure of Integral Environmental Planning - Case Study: Manizales, Colombia, doi:10.17485/ijst/2018/v11i21/122476, Indian Journal of Science and Technology, 11(21) (2018a).

Escobar D., J. Montoya y C. Moncada. Evolution of Urban Accessibility in Manizales, Colombia, 2010 - 2017, doi:10.17485/ijst/2018/v11i18/122745, Indian Journal of Science and Technology, 11(18) (2018b).

Farber, S. y L. Fu. Dynamic public transit accessibility using travel time cubes: Comparing the effects of infrastructure (dis)investments over time, doi: 10.1016/j.compenvurbsys.2016.10.005, Comp., Env. and Urb. Sys., 62 (2017).

Geurs, K. T. y J. Ritsema van Eck. Accessibility measures: review and applications. Evaluation of accessibility impacts of land-use transportation scenarios, and related social and economic impact. RIVM Report, 787, 1-265 (2001).

Geurs, K. T. y J. Ritsema van Eck. Evaluation of accessibility impacts of land-use scenarios: The implications of job competition, land-use, and infrastructure developments for the Netherlands, doi: 10.1068/b12940, Environment and Planning B: Planning and Design, 30(1), 69-87 (2003).

Geurs, K. T. y B. van Wee. Accessibility evaluation of land-use and transport strategies: review and research directions. doi:10.1016/j.jtrangeo.2003.10.005, Journal of Transport Geography, 12, 127-140 (2004).

Grengs, J. Nonwork Accessibility as a Social Equity Indicator, doi:10.1080/15568318.2012.719582, International Journal of Sustainable Transportation, 9(1) 1-14 (2014).

Hansen, W. G. How Accessibility Shapes Land Use, doi:10.1080/01944365908978307, Journal of the American Institute of Planners, 25(2), 73-76 (1959).

Higgs, G., S. Jones, M. Langford y J. Heley. Assessing the impacts of changing public service provision on geographical accessibility: An examination of public library provision in Pembrokeshire, South Wales, doi: 10.1177/2399654417715457, Environment and Planning C: Politics and Space, 36(3), 548-568 (2017).

Ingram, D. R. The Concept of Accessibility: A search for an operational form, doi: 10.1080/09595237100185131, Regional Studies, 5(2), 101-107 (1971).

Kozyrev, V. P. Graph Theory, 10, 489-519 (1972).

Mavoa S., K. Witten, T. McCreanor y D. O'Sullivan. GIS based destination accessibility via public transit and walking in Auckland, New Zealand. doi:10.1016/j.jtrangeo.2011.10.001, Journal of Transport Geography, 20, 15-22 (2012).

Mello, A., y L. Portugal. Um procedimento baseado na acessibilidade para a concepção de planos estratégicos de mobilidade urbana: O caso do Brasil, doi: 10.4067/S0250-71612017000100005, Eure, 43(128), 99-126 (2017).

Moncada, C., S. Cardona y D. Escobar. Saving Travel Time as an Urban Planning Instrument. Case Study: Manizales, Colombia, doi: 10.5539/mas.v12n6p44, Modern Applied Science, 12(6), 44-57 (2018).

Montoya, J., D. Escobar y C. Moncada. Propuesta de ubicación de nuevos centros comerciales, aplicación de un análisis de accesibilidad territorial urbana. Revista Espacios, ISSN 0798 1015, 38(51), 4 (2017).

Nassir, N., M. Hickman, A. Malekzadeh y E. Irannezhad. A utility-based travel impedance measure for public transit network accessibility, doi: 10.1016/j.tra.2016.03.007, Transportation Research Part A: Policy and Practice, 88, 26-39 (2016).

Olsson, M. Functional regions in gravity models and accessibility measures, doi: 10.1515/mgr-2016-0011, Moravian Geographical Reports, 24(2), 60-70 (2016).

Rosero L. Spatial access to health care in Costa Rica and its equity: a GIS-based study, doi: 10.1016/S02779536(03)00322-8, Social Science \& Medicine, 58, 1271-1284 (2004).

Simpson, T. W., T. M Mauery, J. Korte y F. Mistree. Kriging models for global approximation in simulation-based multidisciplinary design optimization, doi:10.2514/3.15017, AIAA Journal, 39(12), 2233-2241 (2001).

Xiao, X., L. Aultman, R. Manning y B. Voigt. The impact of spatial accessibility and perceived barriers on visitation to the US national park system, doi: 10.1016/j.jtrangeo.2018.03.012, Journal of Transport Geography, 68(April), 205-214 (2018).

Xiao, Y., C. Sarkar, y otros 3 autores. Street network accessibility-based methodology for appraisal of land use master plans: An empirical case study of Wuhan, doi:10.1016/j.landusepol.2017.09.013, Land Use Policy, 69, 193-203 (2017).

Zuluaga, J. y D. Escobar. Geomarketing Analysis for Shopping Malls in Manizales (Colombia). Accessibility approach methodology, Revista Espacios, ISSN 0798 1015, 38(21), 20 (2017). 